\title{
Die Nutzung des Internet für den DaF-Unterricht
}

\author{
Angelika Braun
}

Seit September 1996 werden an der Deutschabteilung des Instituts für Moderne Fremdsprachen an der Universität Guadalajara Möglichkeiten der Nutzung der neuen Kommunikations- und Informationstechnologien: E-Mail-Tandem und WorldWideWeb, für den Deutschlernprozeß auf Grundstufenniveau erprobt.

Aufgaben für die Arbeit mit dem WWW, auf deren Darstellung sich der Beitrag beschränkt, wurden zunächst für das Ende des zweiten und für das dritte Semester entwickelt (eine Ausweitung auf höhere Semester ist vorgesehen) und stehen in thematischem Zusammenhang mit Kap. 8-12 des Lehrwerks Sprachbrïk$k e$ (Mebus et al. 1987) ${ }^{1}$. Sie werden in den Gruppen vorbesprochen und erklärt, und auch die Ergebnisse werden, soweit möglich, in den Gruppenunterricht einbezogen. Abgesehen von zwei Einführungssitzungen erfolgt die Bearbeitung der Aufgaben aber außerhalb des Unterrichts und auf freiwilliger Basis. ${ }^{2}$ Die Studierenden, die nicht privat an das Netz angeschlossen sind, haben im Rechenzentrum des Fachbereichs Internetzugang.
Der Beitrag wird zunächst kurz darstellen, welchen Nutzen für den Sprachlernprozeß wir in der Arbeit im Internet sehen, vor allem aber anhand einiger Beispiele unsere Ziele und damit Kriterien für die Entwicklung von Aufgabenstellungen reflektieren.

\subsection{Der Nutzen der Nutzung des Inter-} net beim Lernen (und: Lehren) von Deutsch als Fremdsprache

Die Attraktivität des Internet für das Lernen (und Lehren) von Fremdsprachen liegt zunächst darin, daß es authentische Texte und Informationen ${ }^{3}$ preiswert und aktuell zugänglich macht. Dadurch kann es ein Stück deutschsprachigen Alltag auch in Lernkontexte wie den unseren holen, i.e. in Länder fern von der Zielsprachenkultur:

"Outside of an expensive plane ticket the Internet is the next best thing to being there with its graphics and descriptive information « (Rosen 1996: 3).

Gerade in den Ländern, in denen die Beschaffung von Broschüren, Zeitschriften und Büchern mit erheblichen Kosten

1 Kapitel 8: Arbeitszeit in Deutschland, Lokalgeschichte, Stadtsage; Kapitel 9: Essen und Trinken, Internationale Küche, Kuchenbacken, Wiener Kaffeehauskultur; Kapitel 10: Fachsprachen; Kapitel 11: Stipendium für Deutschland; Kapitel 12: Landwirtschaftliche Produkte, Internationaler Handel.

2 »Didaktisches Einsatzmodell OFF-line / On-line / Off-line« (nach Andreas Lixl-Purcell http: / / www.uncg.edu/ lixlpurc/NetzSpiegel/intro.html).

3 Ich verwende hier und im folgenden diesen Begriff in Abgrenzung lediglich zu »für didaktische Zwecke bearbeitet oder produziert«. Dabei darf nicht außer Acht gelassen werden, daß es sich hier um »virtuelle Authentizität « handelt, die manipulierbar ist und nicht der Realität entsprechen muß. 
verbunden und nur mit zeitlicher Verzögerung möglich ist, und in diesen Ländern vor allem in den Orten außerhalb der großen Metropolen, kommt dem Internet außerdem als Materialfundus eine besondere und in Zukunft sicherlich wachsende Bedeutung $\mathrm{zu}$, indem es geeignet ist, das Wissens- und Informationsgefälle zwischen höher und schwächer entwickelten Regionen zu verringern. ${ }^{1}$ Lehrende und Lernende erhalten über das Internet Zugang zu aktuellen statistischen Daten, zu Alltagstexten wie Kinoprogrammen, Zugfahrplänen und Wetterberichten, zu graphischem Material wie Stadtplänen und Landkarten aber auch zu Karikaturen und Comics oder zu Texten, Bildern und Stücken aktueller Musikgruppen, zu Informationen über Universitäten, Sprachinstitute und Städte, zu aktuellen online-Ausgaben von Tageszeitungen und Zeitschriften sowie zu literarischen oder fachwissenschaftlichen Texten und zu einschlägigen Institutionen und Verbänden, um nur einige der im WWW enthaltenen Textsorten zu nennen. $^{2}$

Wie andere authentische Kommunikationssituationen auch stellt das Internet ein Anwendungsfeld für Lernerstrategien dar (sowohl für Sprachlernstrategien als auch für Sprachverwendungsstrategien, vgl. Tönshoff 1995: 12), fordert und entwickelt darüber hinaus aber auch neue Strategien für Recherche, Selektion und Navigation, die inzwischen Schlüsselqualifikationen für das Berufsleben darstellen. Das Internet ermöglicht Formen selbständigen, autonomen Lernens, das unabhängig von Lehrer und Gruppenunterricht und differenziert nach spezifischen (thematischen und sprachlichen) Interessen sowie nach Leistungsstand der Lernenden stattfinden kann.

Als Medium bietet das Internet die Möglichkeit, verschiedene Formen der Informationsdarbietung durch Bild/Grafik, Ton und schriftlichen Text zu kombinieren, d. h. über multiple Kodierungssysteme und Wahrnehmungsmodalitäten das Lernen zu unterstützen. (Vgl. z. B. Biechele 1996: 750f. und Bayerlein 1996: 729. ) In vielen Fällen läßt es Anfragen, Rückmeldungen, Kritik und Vorschläge zu, ist also interaktiv.

Schließlich ist die (noch?) sehr hohe Motivation der Studierenden zur Arbeit am Computer und besonders mit dem Internet zu nennen. Mark Warschauers empirische Untersuchung zur Nutzung des Computers für Schreiben und Kommunikation ergab,

»that the students overall had a positive attitude toward using computers and that this attitude was consistent across a number of variables, including gender, typing skill and access to a computer at home« (Warschauer 1996: 1).

Fragegeleitete und problemorientierte Aufgabestellungen für die Internet-Re-

1 Die Möglichkeit, über das Ausdrucken von Web-Texten schnell und preiswert authentische und aktuelle oder auch literarische Texte als Unterrichtsmaterial zu gewinnen, bietet sich selbstverständlich generell und auch den KollegInnen, die die Arbeit mit den neuen Medien selbst noch nicht sofort in den Unterricht integrieren wollen oder können. Für die Lehrenden war z. B. von Interesse die Information über die »Entwicklung der Freizeit und der Arbeitszeit von der Antike bis heute«, die der frechverlag im Internet anbietet (http: / / www.frech.de/ fzteil1.html).

2 Über die Server des Instituts für deutsche Sprache (http://www.ids.de), des GoetheInstituts (http:/ / www.goethe.de) und die Internet-Seiten von Andreas Lixl-Purcell (http:/ / www.uncg.edu/ lixlpurc/german/) und von Herbert J. Holzinger (http:/ / www.uv.es/ holzinge/comp.html\#Internet) sind über aktualisierte Links zahlreiche DaF-Ressourcen im Internet zu finden. 
cherche, die entdeckendes Lernen initiieren, können diese Motivation weiter erhöhen. Der Neuigkeitseffekt, der Lerner begeistert sogar mit Lernprogrammen traditionellster Art arbeiten läßt, sofern sie über den Computerbildschirm dargeboten werden, wird sich sicherlich bald abnutzen, nicht aber das Interesse an wirklich authentischen Materialien im Web und die Neugierde beim Suchen von Informationen, eine dem Lernen inhärente und förderliche Haltung.

\subsection{Suchtechniken}

Das Internet und die darin enthaltenen Informationen werden von den Studierenden inzwischen bereits am zweithäufigsten genannt, wenn wir fragen, wo sie ihre Deutschkenntnisse bereits jetzt auBerhalb des Unterrichts anwenden können. ${ }^{1}$ Nur ca. $20 \%$ der Teilnehmer verfügten aber bereits vor Beginn des Projekts über erste Erfahrungen bei der Nutzung der Neuen Medien, nur vereinzelt handelt es sich dabei um routinierte Surfer. Aus dieser Konstellation leitet sich als erstes (instrumentelles) Ziel unseres Projekts $a b$, die Studierenden mit dem neuen Medium und den wichtigsten Techniken im Umgang mit ihm vertraut zu machen. ${ }^{2}$

Über ein Aufgabenblatt zu der deutschen Leitseite des Suchdienstes Yahoo.de (http://www.yahoo.de), eines der
»Pfadfinder« durch die unüberschaubare Menge von Informationen im Internet $^{3}$, werden in einer Einführungssitzung Techniken zum Auffinden von Informationen vermittelt, z. B. die Suche nach einem oder mehreren Stichworten (Suche starten), Möglichkeiten zur Reduktion der manchmal erschlagenden Menge der Suchergebnisse (advanced search) sowie die Nutzung der sich immer weiter verzweigenden Kategorien und Subkategorien. Gleichzeitig wird mit dieser Seite die Struktur des Internet als die eines nichtlinearen Hypertextes, d.h. als eines Verbunds von Einzeltexten, erklärt, die durch markierte Links (in unserem Beispiel die Kategorien und Subkategorien) netzartig miteinander verknüpft sind. Die Links leiten als »active footnotes « (Fidelman 1996: 5) den Nutzer durch Anklicken automatisch zu einem anderen Text, der seinerseits durch Links auf andere Texte verweist. Am Beispiel der Stadt Hameln werden die Lernenden in die Systematik der Internet-Adressen eingeführt (s. Abb. 1 im Anhang, Einleitung), die es in vielen Fällen ermöglicht, Städte, Universitäten, Institutionen, Zeitungen etc. direkt und ohne den (Um-)Weg über einen Suchdienst $\mathrm{zu}$ finden. ${ }^{4}$

Exemplarisch wird der Aufbau einer solchen Leitseite erklärt, und die Studierenden sammeln erste Erfahrungen sowohl mit gezieltem als auch unkontrolliertem

1 An erster Stelle steht die Kommunikation mit deutschen Studierenden, die über den Verein für Interkulturellen und Internationalen Austausch (VIA e.V.) für ein Praktikum nach Guadalajara kommen und bei unseren Studierenden wohnen. An dritter Stelle werden deutschsprachige Touristen in Guadalajara und den Badeorten an der Pazifikküste genannt, an vierter Stelle stehen deutschsprachige Zeitungen oder Zeitschriften.

2 Vgl. auch Carolyn Fidelmans Language Professional's Guide to WWW (http: / / agoralang. $\mathrm{com} /$ calico/webarticle) und den »Online-Guide« der University of Victoria: http:// webserver.maclab.comp.uvic.ca/onlineguide/welcome.html.

3 Daneben sind empfehlenswert: webcrawler (http:/ / www.crawler.de), infoseek (http: / / www.infoseek.com), DINO (http://www.dino-online.de), Lycos (http://www.lycos. de), altavista und für die Suche nach Textteilen (Liedzeilen!!) oder Information über Personen hotbot (http:/ / www.hotbot.com). 
Anklicken von Buttons und anderen Links. Hierbei werden die Möglichkeiten, aber auch die Probleme des Surfens im Internet deutlich: Die Möglichkeit, durch gezielte Suche rasch die gewünschten Ergebnisse zu erhalten, die Gefahr, sich bei ungezielter Suche im Web zu verheddern, aber auch die Möglichkeit, dabei auf interessante Informationen $\mathrm{zu}$ stoßen, die man gar nicht vermutet (und daher: gesucht) hätte. Die Aufgabe, Suchwege selbst zu finden bzw. sich bewußt zu machen, wiederholen sich bei den meisten Aufgabenblättern und verfolgen das Ziel, die Studierenden zur selbständigen Orientierung im Web zu befähigen. Dieses Vorgehen wurde der Möglichkeit vorgezogen, Internet-Adressen auf dem Aufgabenblatt oder über Links auf einer eigenen Home Page vorzugeben ${ }^{1}$, wie es bei Web-Aufgaben üblich ist, die von Dozenten an USamerikanischen, kanadischen oder australischen Universitäten entwickelt wurden, denen wir viele Anregungen für Aufgabenstellungen und Tips zu interessanten Materialien im Web verdanken. $^{2}$

\subsection{Eine Vielfalt von Materialien für eine Vielfalt thematischer Interessen}

Auch diejenigen Studierenden, die bereits vor Beginn des Projekts über Erfahrung mit dem Internet verfügten, hatten es noch nicht im Zusammenhang mit dem Deutschunterricht genutzt. Ein zweites Ziel des Projekts besteht deshalb darin, den Teilnehmern einen Einblick in die im Internet zugänglichen Textsorten mit Bezug zu deutschsprachigen Ländern oder zur deutschen Sprache zu geben, ihre Neugierde diesen Materialien gegenüber zu wecken oder zu verstärken und sie zu ermutigen, von diesen nach Maßgabe ihrer Interessen Gebrauch zu machen.

Neben den Materialien, auf die die abgebildeten Aufgabenblätter verweisen (Abb. 1-5 im Anhang), wurde mit Informationen über deutsche Verkehrszeichen und -regeln, Stefan Zweig, Wiener Kaffeehäuser, deutsches Bier und einem Aufruf zum Boykott mexikanischen Kaffees aus Chiapas gearbeitet. Musikinteressierte stellten ihren KommilitonInnen die Pop-Gruppen Die Prinzen und Die fantastischen Vier vor. Für den weiteren Verlauf des Semesters sind Aufgaben zum Kursangebot des Goethe-Instituts, zum Sti-

4 Der Spiegel z. B. ist unter http: / / www.spiegel.de, die Universität Tübingen unter http: / / www.uni-tuebingen.de, die Bundesregierung unter http:/ / www.bundesregierung.de, die Stadt Hamburg unter http: / / www.hamburg.de zu finden. Die »Endung «.de meint Deutschland, .ca Canada etc., .com kommerzielle Anbieter, .edu Adressen aus dem Bereich Bildung etc.

1 Für unser Vorgehen spricht auch der Umstand, daß Internet-Adressen eine nur geringe Halbwertzeit haben, d.h. sich relativ schnell ändern, und die »Umzüge« nur für eine relativ kurze Zeitspanne angezeigt werden. Wenn wir den Studierenden nur die aufzusuchende Adresse angeben, nicht aber zeigen, wie sie sie selbst finden können, wird die gegebene Information schnell obsolet.

2 Zum Beispiel Peter Gölz'Web-Exercises (http://web.UVic.ca/german/149/web-exercises.htm), Andreas Lixl-Purcells Netzbox Grundstufe (http:/ / www.uncg.edu/ lixlpurc/ publications / NetBo2.html) und Netzspiegel (http: / / www.uncg.edu/ lixlpurc/publications/NetzSpiegel), Inge DiBellas Web-Exercises (http://wsrv.clas.virginia.edu/ iad4c/webex.html), die Aufgaben des Goethe-Instituts zu Artikeln aus dem Jugendmagazin jetzt-online (http:/ / www.goethe.de/z/jetzt/deindex.htm) und Manfred Prokops Zusammenstellung in Using the Web for Language Exercises and Readings of Authentic Texts (http:/ / www.ualberta.ca/ german/present.htm). 
pendienprogramm des $\mathrm{DAAD}, \mathrm{zu}$ online-Zeitungen und -Zeitschriften, $\mathrm{zu}$ Bio-Produkten und Bio-Anbaumethoden sowie zum Außenhandel der Bundesrepublik vorbereitet. Beim Statistischen Bundesamt (http:/ / www.statistik-bund. de) wurden aktuelle Daten über den Getränkekonsum in Deutschland und über die durchschnittliche Arbeitszeit in verschiedenen Branchen per E-Mail angefordert. Die aktuelle Tabelle des Bundesministeriums für Ernährung, Landwirtschaft und Forsten zum Gebrauch von Getränken in Deutschland erhielten wir tatsächlich bereits am nächsten Tag per Fax.

Nur einige dieser Aufgaben sollen, konventionellen Hausaufgaben vergleichbar, von allen Studierenden bearbeitet werden, meist müssen diese selbst entscheiden, ob sie das Thema interessiert und ob sie bereit und in der Lage sind, die für seine Bearbeitung erforderliche Zeit aufzubringen. Bei nahezu allen Aufgabenblättern werden den Studierenden außerdem Optionen für die Weiterarbeit gegeben, deren Ergebnisse in der Lerngruppe präsentiert werden können. (s. Abb. 1: 14f., Abb. 2, Abb. 3: 4, Abb. 4: 9-11, 18, Abb. 5). Mit den beiden Prinzipien $» F r e i-$ willigkeit « und »Angebote zur Weiterarbeit « versuchen wir deutlich zu machen, daß das Internet gerade für spezifische Lernerinteressen, die nicht immer und nicht alle im Kursprogramm berücksichtigt werden können, eine interessante Option darstellt. Darunter fallen u. a. Bereiche der Alltagskommunikation in deutschsprachigen Ländern, die in Übereinstimmung mit dem von den Autoren des Lehrwerks Sprachbrücke vertretenen Ansatz im Kursunterricht weitgehend ausgespart bleiben (Rall 1990: 15f.), viele Studierende aber - und nicht nur diejenigen, die in näherer Zukunft eine Reise nach Deutschland planen - dennoch stark interessieren.

\subsection{Textverarbeitungsstrategien: Such- und Lesetechniken}

Angesichts der ungeheuer großen Menge von Texten und der Textmengen im Web ist es nicht nur wichtig, daß die Studierenden Suchtechniken beherrschen und selbständig - kursbezogene und kursunabhängige - Fragestellungen entwickeln, sondern auch, daß sie sich von langen und für sie sprachlich schwierigen Texten nicht entmutigen lassen. Die psychische Disposition der Lernenden, sich auf diejenigen Textteile zu konzentrieren, die sie verstehen können, und Toleranz gegenüber dem Nochnicht-Verstehen anderer Teile zu entwickeln, ist unserer Erfahrung nach zentral für ein erfolgreiches Arbeiten mit den Materialien des Internet bereits in der Grundstufe.

"A text is never stoo difficult: it depends what you want the students to do with it and how you structure this activity« (Di Bella 1996). ${ }^{1}$

Das Internet fordert und fördert die Anwendung von Lesestrategien, die dem Sprachstand und Informationsbedürfnis der Nutzer entsprechen und die im konventionellen Unterricht mit »HäppchenTexten « so schwer zu vermitteln, aber so wichtig für den Umgang mit allen authentischen Texten sind. ${ }^{2}$ Beispiele für

1 D.h. auch, sich zu fragen, in welcher Sprache die Aufgaben gestellt und die Antworten gefordert werden. Bei rein inhaltsbezogenen Aufgabenstellungen ist das Vorgehen von Katherine Munro (www.gil.com.au/ kmunro/ger-ex.htm), die dafür die Muttersprache der Lerner wählt, durchaus sinnvoll.

2 Interessant wäre sicher, der Frage nachzugehen, wie das »flächige, nicht lineare« Lesen schriftlicher Texte in multimedialer Umgebung vor sich geht (vgl. Schmitz 1996). 
entsprechende Aufgabenstellungen - mit vorbereitenden Aufgaben und Links zu Worterklärungen, weiterführenden Informationen und Tips für die Anwendung elementarer Lesetechniken - zu Artikeln aus dem Jugendmagazin jetzt-online sind auf dem Server des Goethe-Instituts zu finden (http://www.goethe.de/ $\mathrm{z} /$ jetzt/deindex.htm).

Abb. 1: 2 und 3 zielen auf die Aktivierung des Vorwissens der Studierenden, das das selektive Verstehen des relativ langen und schwierigen Textes organisiert, d.h. das gezielte Suchen nach zu erwartenden Informationen (Lage, Einwohnerzahl, Industrie, Touristenattraktionen, Geschichte z. B.) und deren Auffinden ermöglicht. Dieses Vorgehen wiederholt sich bei der Erschließung der Rattenfängersage, deren Inhalt den Studierenden bekannt ist (Abb. 1: 5-9) und bei der Suche nach Informationen zu Heinrich Hoffmann (Abb. 3: 3).

Andere Aufgabenstellungen erfordern kursorisches Lesen bzw. globales Leseverstehen (z. B. - mit Hilfe der Zeichnungen - die Erschließung des Textes vom Suppenkaspar, Abb. 3: 1). Nur in seltenen Fällen und aufgrund einer bewußten Entscheidung (Abb. 4: 18) müssen Texte tatsächlich im Detail verstanden werden.

Wo immer möglich, werden die Studierenden implizit oder explizit angehalten, unbekannte Wörter zu erschließen. Die meisten Kategorien und Sub-Kategorien, mit denen der Suchdienst yahoo.de arbeitet, und einige Zutaten in den Rezeptsammlungen (Abb. 4: 6 u. 16) sind Internationalismen und damit auch für Grundstufenlerner leicht verständlich, die Adjektive im Text vom Suppenkaspar erklären sich über die Zeichnungen (Abb. 3: 2), viele Verben in Rezepten sind über Kollokationen und ihre Position im Text verständlich (Abb. 4: 17). ${ }^{1}$

\subsection{Sprachen lernen im Internet - eine (noch?) ungenutzte Möglichkeit}

Colonel Craig eröffnet die Sektion Learning the German Language in seiner Sammlung von WWW Links for German (http: / / www.isu.edu/nickcrai/german.html) mit den Worten:

»Ach! The Internet ist not a great place for language learning, if you're doing that alone-But if that's your only option at the moment, here are few sites that will help«.

Tatsächlich ermöglichen Sprachlernprogramme im Web zwar potentiell autonome Lernformen, entsprechen aber häufig nicht den didaktisch-methodischen Standards, die an Aufgabenstellungen im interkulturellen kommunikativen Fremdsprachenunterricht angelegt werden. Abb. 6 zeigt ein - durchaus nicht außergewöhnliches - Beispiel für dekontextualisierte Übungen zu Wortschatz, Morphologie und Syntax. Übungen dieser Art nutzen weder verschiedene Kanäle für die Informationsdarbietung noch die Interaktivität des Mediums: Die LernerInnen können die Aufgaben nicht am Computer lösen und bekommen weder unmittelbares Feed Back noch Hilfestellungen. Die einzige Innovation besteht darin, daß sie aufgefordert werden, ihre Lösungen per E-Mail an den Lehrenden $\mathrm{zu}$ schicken. Ich denke, es ist für nichtkommerzielle Anbieter zu aufwendig, gute interaktive Aufgaben zu programmieren, die diese Standards erfüllen. ${ }^{2}$ Deshalb werden die miserablen Resultate von PC-Programmen für das Fremdspra-

1 Diesem grundlegenden Prinzip für das Lesen, auch in der Fremdsprache, widerspricht leider die Anlage der im texthaus des GI Mailand (http://www.webcom.com/ texthaus) aufbereiteten Texte, in denen dem Nutzer durch Links suggeriert wird, Erklärungen für ohne weiteres erschließbare Wörter (z. B. Internationalismen) zu suchen. 
chenlernen bei SODIS ${ }^{1}$, das lediglich 3\% der Programme als »empfehlenswert« beurteilt, von den Lernprogrammen im Internet meines Erachtens sogar noch übertroffen. Aber wenn wir den Studenten Wege zeigen, wie sie ihre Sprachkenntnisse nicht nur unabhängig vom Lehrer und dem Unterricht, sondern auch unabhängig von Autoren von Lernprogrammen, d.h. mit authentischen Texten verbessern können, ist das Internet eine unerschöpfliche Quelle auch von Sprachlernsituationen und geeignet, nicht nur die Sprach-, sondern auch die Lernkompetenz der Studierenden zu fördern (Jenfu 1995).

Auf das Potential der Materialien im Web für die sprachliche Weiterentwicklung der Lerner verwiesen schon die am Ende des vorigen Abschnitts erwähnten Aufgaben, die darauf zielen, bekannte Inhalte in ihrer fremdsprachlichen Repräsentation wiederzuerkennen.

Am deutlichsten wird diese Möglichkeit beim Thema Rezepte genutzt. Das starke Interesse einiger Studierender an diesem Thema einerseits und andererseits die Unmöglichkeit, es in extenso im Unterricht zu behandeln, empfehlen es in besonderem Maße für die individuelle Kompetenzerweiterung durch die auto- nome Arbeit mit dem Internet. Die Aufgabenstellung suggeriert Schritte für das Vorgehen: vom Bekannten (Abb. 4: 16, identifizieren/verstehen) zum Unbekannten (Abb. 4: 17, deduzieren, Hypothesenbildung) im Bereich der Wortschatzerweiterung.

Ein weiteres Ziel dieser Aufgabe ist die Nutzung der im Internet enthaltenen Rezepte für das Verfassen eigener Rezepte auf deutsch (Abb. 5), d.h. als Modelle für die Textproduktion. Durch die Einbindung der Rechercheergebnisse in den Gruppenunterricht bzw. in den hier nicht dargestellten Projektteil E-Mail-Tandem wie auch durch das Versenden einer Nachricht an den Autor oder Verantwortlichen der jeweiligen Seite im Internet per E-Mail werden durch die Arbeit im Web neben dem Leseverstehen und der Wortschatzerweiterung auch die produktiven Fertigkeiten Schreiben und Sprechen gefördert. ${ }^{2}$

Eine weitere, von uns allerdings noch nicht erprobte Möglichkeit der Nutzung des Web für Kommunikation, die zwar schriftlich stattfindet, aber wesentliche Merkmale mündlicher Kommunikation aufweist, stellen Diskussionsgruppen und sogenannte $\mathrm{MOOs}^{3}$ dar, virtuelle Räume, in denen sich verschiedene Teil-

2 Diese These wird illustriert durch die Tatsache, daß Johannes Schumann im Internet 1000 Kreuzworträtsel anbietet, die aber nicht am Computer gelöst werden können, während sich Detlef Biehn, GI New York, in einem m. E. zwar immer noch unbefriedigenden, im Vergleich aber weit besseren Programm auf zwei Rätsel beschränkt und betont, daß die Gestaltung dieser Rätsel »sehr komplex und mit erheblichem Zeitaufwand verbunden « war (http://www.goethe.de/uk/ney/kreuzwort/dekwr.html). Aufwendigere Programme (z. B. der Sprachkurs auf der in Anm. 14 erwähnten Seite des GI Mailand) sind nur gegen Entgelt zu nutzen.

1 Das Landesinstitut für Schule und Weiterbildung, Beratungsstelle für Neue Technologien, Soest, publiziert regelmäßig den SODIS-Beurteilungsbogen, in dem PC-Programme in didaktisch-methodischer Hinsicht bewertet werden. 1996 wurden nur etwa 3\% aller 3.798 Programme (davon 70 für DaF) als »empfehlenswert« bewertet. Vgl. auch Bayerlein (1996) und Hahn (1995). Die dort angestellten Überlegungen sind weitgehend auf Lernprogramme im Internet übertragbar.

2 Vgl. dazu auch den interessanten Vorschlag von Lauren Rosen (http:/ / www.Iss.wisc. edu/Iss/lang/), Studenten in Lerngruppen unterschiedlicher Muttersprachen zweisprachige Web-Seiten gestalten zu lassen. 
nehmer zu einer verabredeten Zeit treffen und miteinander kommunizieren. Noch in den Anfängen stecken Versuche, Listen von Artikelüberschriften, die man über den Suchdienst Altavista nach Eingabe eines Suchbegriffs erhält, als Ersatz für Konkordanzprogramme zu nutzen. ${ }^{1}$

\subsection{Beispiel für Einsatz eines Lernpro- gramms.}

Es ist sicher deutlich geworden, daß es uns bei unserem Projekt in erster Instanz um die Arbeit mit authentischen Materialien im Web geht, die in anderer Form nicht oder nur schwer zugänglich zu machen sind. Dennoch geben wir den Studierenden auch Informationen darüber, unter welchen Adressen sie im Internet Sprachlernprogramme finden können die von den Studierenden übrigens (weil sie ihren Lerntraditionen entgegenkommen??) durchweg positiver beurteilt werden als von den Lehrenden - und erarbeiten dazu in Einzelfällen sogar Aufgabenblätter.

Ein Beispiel dafür ist Abb. 7 zur Arbeit mit dem einem Reisesprachführer verwandten Sprachlernprogramm des Servers travlang (http://www.travlang. com), der den Studierenden nicht nur Informationen für eine Deutschlandreise (Zugverbindungen, Wetter, Städte) gibt und ein Wörterbuch und eine Grammatik anbietet, sondern auch Redemittel, hier: für die Kommunikationssituation im Restaurant (bestellen, bezahlen), zur Verfügung stellt. Bei der Aufgabenstel- lung haben wir vor allem auf die Anwendung metakognitiver Lernerstrategien für die Planung, Kontrolle und Evaluierung des Lernprozesses Wert gelegt (O'Malley/Chamot 1990: 144), d.h. die bewußte Handhabung des Programms in Bezug auf Themenwahl, Protokoll der Ergebnisse, regelmäßige Wiederholung etc.

\subsection{Weitere Perspektiven}

Eine Umfrage zur Auswertung des Projektes ist für Ende des Semesters vorgesehen. Es ist aber festzuhalten, daß es trotz mehrerer organisatorischer Schwierigkeiten (kein Platz an Rechnern, lange Ladezeiten zu den Hauptnutzungszeiten, Mängel bei Hard- und Software im Rechenzentrum etc.) allen Beteiligten großen Spaß macht und die Berichte von den Rechercheergebnissen im Unterricht Multiplikatoreneffekt hatten: Inzwischen haben sich fast alle Studierende in den Pilotkursen Zugangsmöglichkeiten besorgt, die Zahl derer, die die Aufgabenblätter bearbeiten, steigt, und die Studierenden anderer Semester fordern die Implantierung des Projekts auch in ihren Kursen ein.

Wir würden uns freuen, wenn sich dieser Effekt auch unter den KollegInnen einstellen würde und wir zu einem Austausch von Informationen über interessante Materialien und über die Entwicklung und Evaluierung von Aufgaben für die Internet-Recherche kommen könnten.

$3 »$ MOO steht für MUD Object Oriented und MUD für Multi User Domain oder Multi-User Dungeon « (Brammerts / Little 1996: Anm. 2). Vgl. diese Arbeit und Lonnie Turbee in http:/ / web.syr.edu / Imturbee auch zu den Arbeitsmöglichkeiten mit MOO.

1 Zur Arbeit mit Konkordanzprogrammen siehe Rüschoff (1995). 


\section{Literatur}

Alle Literaturangaben im Internet wurden kurz vor Drucklegung des Manuskripts überprüft und nötigenfalls aktualisiert. Wie in Anmerkung 10 erwähnt, tragen sie aber das Risiko der geringen Halbwertzeit in sich. Hilfreich beim Aufspüren sind die in Anmerkung 5 angegebenen bislang stabilen Server, die links zu fast allen angegebenen Seiten enthalten.

Bayerlein, Oliver: "Versuch einer Übungstypologie für computergestützte Multimedia-Sprachkurse«, InfoDaF 23, 6 (1996), 726-736.

Biechele, Barbara: »Bilder als Kommunikate und Lernmedien im Fremdsprachenunterricht DaF«, InfoDaF 23, 6 (1996), 746757.

Biehn, Detlef: Goethe-Institut New York, http: / / www.goethe.de/uk/ney/kreuzwort/dekwr.html. (21.11.1997).

Brammerts, Helmut; Little, David: Leitfaden für das Sprachenlernen im Tandem über das Internet. Bochum: Universitätsverlag Dr. N. Brockmeyer, 1996 (Manuskripte zur Sprachlehrforschung 52).

Colonel Craig: WWW Links for German. http: / / www.isu.edu/ nickcraig/german.html, (21.11.1997).

Di Bella, Inge A.: Teaching with the WWW. Guiding Principles for creating Teaching Units. http: / / wsrv.clas.virginia.edu / $\sim$ iad4c/www.html. (21.11.1997).

Fidelman, Carolyn: Language Professional's Guide to WWW. http:9/www.agoralang. com/calico/ webarticle (21.11.1997).

frechverlag: Die Entwicklung der Freizeit und der Arbeitszeit von der Antike bis heute. http: / / www.frech.de/ fzteil1.html (21.11. 1997).

Hahn, Martin: »Medienintegration - Multimedia - Interaktive Medien«, Zielsprache Deutsch 26, 3 (1995), 122-135.

Jenfu, Ni: »Lernkompetenzförderung - ein legitimes Anliegen des Fremdsprachenunterrichts«, Neusprachliche Mitteilungen 48 (1995), 148-157.

Lixl-Purcell, Andreas: »Hinweise zur Didaktisierung«. In: Netzspiegel: Deutsche Landeskunde im Internet. http://uncg. edu / lixpurc/NetzSpiegel/ Netzspiegel. html. (21.11.1997).

Mebus, Gudula; Pauldrach, Andreas; Rall, Marlene; Rösler, Dietmar: Sprachbrücke 1. Stuttgart: Klett, 1987.
O'Malley, J. Michael; Charmot, Anna Uhl: Learning Strategies in Second Language Acquisition. Cambridge; New York: Cambridge University Press, 1990.

Prokop, Manfred: Using the Web for Language Exercises and Reading of Authentic Texts. http:/ / ww.ualberta.ca/ german/ present.htm. (21.11.1997).

Rall, Marlene: Sprachbrücke 1, Handbuch für den Unterricht. Stuttgart: Klett, 1990.

Rosen, Lauren: Teaching with Web. http:/ / polyglot.Iss.wisc.edu / Iss / lang / teach. html. (21.11.1997).

Rüschoff, Bernd: »Technologiegestütztes Lernen und innovative Formen der Wortschatzarbeit «, Der fremdsprachliche Unterricht Englisch 1 (1995), 49-52.

Schmitz, Ulrich: Schriftliche Texte in multimedialen Kontexten. http:/ / www.linse.uniessen.de/papers/schriftl_texte.htm. (21. 11.1997).

Tönshoff, Wolfgang: »Fremdsprachenlerntheorie", Fremdsprache Deutsch, Sondernummer 1995, 4-15.

Turbee, Lonnie M.: MOO for foreign language learning and teaching. http:9/ web.syr. edu/ Imturbee/ (21.11.1997).

Warschauer, Mark: Motivational aspects of using computers for writing and communication. Second Language Curriculum Center, University of Hawai'i, 1996.

Zimmer, Hubert D.: »Gedächtnispsychologische Aspekte des Lernens und Verarbeitens von Fremdsprache «, InfoDaF 15, 2 (1988), 149-163.

\section{Anhang}

Abb. 1

Name:

Hameln hat - wie die meisten anderen deutschen Städte - eine Seite (homepage) im Internet. Du findest sie unter der Adresse http://www.hameln.de

Besuche die Stadt Hameln im Internet!

1) Wie bekommst du Informationen über Hameln?

2) Welche Informationen erwartest du?

3) Suche die Informationen und notiere sie bitte auf der Rückseite. 
4) Was siehst du oben links?

(auf deutsch heißt das Stadtwappen) Was sagt dir das über die Stadt?

5) Was siehst du oben rechts? Wie heißt diese Figur auf spanisch?

6) Suche ihren Namen auf deutsch:

7) Was weißt du über die Geschichte dieser Figur? Versuche, dich zu erinnern!

8) Wo im Text findest du ihre Geschichte Notiere bitte die Überschrift:

9) Lies jetzt den Text und versuche, den Inhalt wiederzuerkennen, auch wenn du nicht alle Wörter verstehst.

10) Druck den Text, denn wir wollen ihn im Unterricht besprechen.

11) Hausaufgabe: Markiere 5 Sätze, die du verstehst, weil du die Geschichte kennst. Es ist nicht nötig, daß du alle Wörter verstehst.

14) Welche andere deutsche Stadt möchtest du besuchen?

15) Besuche die Stadt und bereite eine schriftliche Präsentation für die Gruppe vor (1 Seite). Versuche nicht, den ganzen Text detailliert zu verstehen, sondern suche allgemeine Informationen und nur eine »Spezialität« deiner Stadt.

Importante: ¡No trates de comprender toda la información dada en el Internet! ¡Trata de adoptar siempre la actitud de buscar algo! ¡Trata de pensar antes: ¿Qué es lo que esperas obtener? y verifica tus hipótesis!

\section{Abb. 2 Name:}

Hast du Hunger und Durst? - Dann besuche doch - virtuell - ein Restaurant in Deutschland, Österreich oder der Schweiz und bestelle ein opulentes Menü.

Suchweg: yahoo.de, dort schreiben: Speisekarte, dann wählen: Alta-Vista-Web-Sites Dort findest du eine lange Liste mit Restaurants. Ein Klick zeigt dir ihre Speisekarte.
1. In welchem Restaurant hast du gegessen?

2. Wo ist das Restaurant (Stadt und Adresse)

3. Wann ist das Restaurant geöffnet?

4. Ist das Restaurant auf bestimmte Gerichte spezialisiert?

5. Was hast du gegessen?

6. Was hast du getrunken?

7. Was hat alles zusammen gekostet?

8. Hat das virtuelle Essen geschmeckt und bist du satt geworden?

9. Kannst du einen Besuch in diesem Restaurant empfehlen? -

Warum (nicht)?

Wenn du ein Wort wissen möchtest, das du im Wörterbuch nicht findest, kannst du uns per E-Mail fragen.

Wenn dich das Thema »Speisekarten « interessiert, kannst du die Speisekarten-Seite (yahoo.de - schreiben: Speisekarte - der erste Eintrag) besuchen und für die Gruppe einen Bericht vorbereiten.

\section{Abb. 3 Name:}

Weißt du, was ein Suppenkaspar ist?

1. Suche in yahoo.de Informationen. Klick auf Suppen-Kaspar (»Suppern« ist leider falsch geschrieben!)

1.1. Schau die Zeichnungen zu der Geschichte an - Was passiert mit Kaspar?

1.2. Warum? - (Die Antwort gibt dir der Text)

1.3. Was ist also die »Moral« von der Geschichte?

1.4. Gibt es solche Geschichten auch für mexikanische Kinder? 


\section{Wortschatzerweiterung:}

Suche die Wörter im Text, die Kaspars Zustand beschreiben (vor allem Adjektive!) am 1.Tag

$$
\text { am 2.Tag }
$$

.

3. Inhaltliche Erweiterung:

3.1. Gehe zurück zu der Yahoo-Liste und versuche in den anderen Seiten herauszufinden:

Wie heißt der Titel des Buches, in dem die Geschichte vom Suppen-Kaspar steht?

\subsection{Wie heißt der Autor?}

\subsection{Wann hat der Autor gelebt?}

3.4. Welche anderen Geschichten stehen in demselben Buch?

3.5. Welche von ihnen handelt von einem Mädchen?

Gehe zurück zu yahoo.de und suche weitere Informationen zu dem Autor:

3.6. Was war er von Beruf?

3.7. Welche Pseudonyme hat er sich gegeben?

3.8. Welche davon haben mit seiner Tätigkeit als Kinderbuchautor zu tun?

4. Möchtest du noch eine andere Geschichte lesen und in der Gruppe vorstellen?

\section{Abb. 4 Name:}

1. Wie finden wir im Internet Rezepte auf deutsch?

2. Yahoo zeigt dir jetzt 3 Kategorien. Unter welcher Kategorie findest du Rezepte zum Thema »Essen«?

Klick auf diese Kategorie. Yahoo zeigt dir jetzt eine Liste von Rezeptsammlungen, in denen du Rezepte suchen kannst.
3. Was sagen dir die Namen der Sammlungen? Schreibe mindestens je 1 Beispiel für die folgenden Gruppen:

1) Rezeptsammlung einer Person:

2) Die Sammlung hat auch links zu anderen Rezept-Seiten:

3) Spezialrezepte (mit speziellen Zutaten, zu einem speziellen Zweck):

4) Rezepte aus einem anderen Land:

5) Rezepte aus einer bestimmten Region Deutschlands:

6) deutsche Rezepte allgemein:

7) internationale Rezepte:

Besuche bitte Das Kochbuch im Internet

4. Wieviele Rezepte enthält diese Sammlung?

5. Wie heißt der Autor dieses Kochbuchs? .............

6. Die Rezepte sind in Gruppen geordnet. Lies bitte die Liste und überlege:

Welche Wörter oder Wortteile verstehst du schon oder kannst du deduzieren?

7. Welche kennst du noch nicht? Schreib die Wörter auf und suche ihre Bedeutung im Wörterbuch:

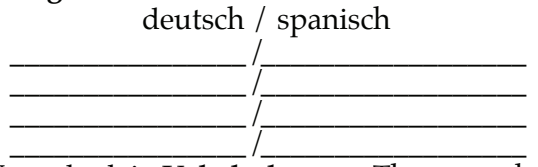

(Wenn du dein Vokabular zum Thema noch mehr erweitern möchtest, kannst du die Aufgaben 6 und 7 an einem anderen Tag mit der Liste in der Rezeptdatenbank bearbeiten)

8. Markiere deine Wünsche für unser gemeinsames Essen!

9. Schau dir noch ein anderes Kochbuch an. Ich habe angesehen. Welches gefällt dir besser und warum? 
10. Hast du noch eine andere Rezeptsammlung im Internet gesehen? Schreibe die Adresse hier auf:

Möchtest du wissen, was die Deutschen für "Mexikanische Küche" halten?

Möchtest du einem Deutschen ein mexikanisches Gericht erklären?

Möchtest du später in Deutschland Mexikanisch kochen und auf dem Markt Zutaten kaufen?

Möchtest du dein Vokabular zum Verstehen von Rezepten erweitern?

Dann helfen dir Rezepte für Mexikanische Gerichte im Internet.

11. Findest du im Kochbuch im Internet mexikanische Gerichte? (Tip: das Stichwortverzeichnis nach der Liste!)

Die Stichworte heißen:

a)

b)

c)

12. Wähle ein Stichwort und schau dir die Liste mit mexikanischen Gerichten an.

13. Was meinst du - sind das »typisch mexikanische« Gerichte? Kennst du sie?

14. Wie kannst du alle diese Gerichte zusammen sehen?

15. Lies ein paar Gerichte und notiere mindestens 6 Vokabeln für Zutaten, die du noch nicht kanntest, die du aber verstehst:

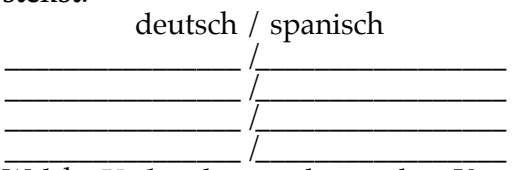

16. Welche Verben kannst du aus dem Kontext verstehen? (Überlege: Was macht man z. B. mit Tomaten: lavar, picar, asar, hervir, ... / Steht das Verb am Anfang, in der Mitte, am Ende des Rezepts?). Schreibe mindestens 6 Verben und deine Hypothesen über ihre Bedeutung:

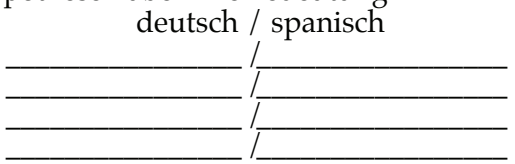

17. Welches Gericht möchtest du genau verstehen (und vielleicht kochen)?

(Wenn du uns eine Mail mit dem Namen schickst, drucken wir es dir aus.)
Abb. 5

1. Erkläre deinem Tandem-Partner dein Lieblingsgericht!

1.1. Welche Zutaten brauchst du? Schreib eine Liste auf Deutsch!

(Prüfe in der Rezeptdatenbank, ob es alle Zutaten in Deutschland gibt. Wenn nicht, kann man dein Gericht dort nicht kochen: Du mußt ein anderes Gericht auswählen!)

1.2. Beschreibe die Zubereitung. Benutze das Kuchenrezept in Sprachbrücke und die Rezeptsammlungen im Internet als Modell.

1.3. Schicke uns dein Rezept per E-Mail, damit wir es korrigieren können.

1.4. Schicke das korrigierte Rezept per EMail an deinen Tandem-Partner.

2. Wenn du möchtest, kannst du das korrigierte Rezept auch an eine Rezeptsammlung im Internet schicken - z. B. an die RezeptDatenbank oder das Internet-Kochbuch. Mal sehen, ob sie es in ihre Sammlung aufnehmen.

3. Frage deinen Tandem-Partner nach seinem Lieblingsgericht.

3.2. Suche das Rezept in den Rezeptsammlungen im Internet! (Wenn du es nicht findest, schick uns eine Mail - vielleicht haben wir mehr Glück!)

3.3. Möchtest du es probieren?

Abb. 6

Übung 3

Ersetzen Sie die Subjekte in diesen Sätzen mit den Wörtern in der Klammer (=parenthesis).

Beispiel:

Wir geben es dir morgen. (sie, Singular)

Sie gibt es dir morgen.

1. Sehen Sie es? (er)

2. Wann treffen Sie ihn? (du)

3. Ich nehme den Anzug. (er)

4. Ich empfehle das Schnitzel. (er)

5. Ich lese jeden Morgen die Zeitung. (er)

Setzen Sie das Modalverb in die Sätze ein. Benutzen Sie die gleiche Zeit!

Beispiele:

Ich stehe immer früh auf. (müssen)

Ich muß immer früh aufstehen. 
Ich besuchte ihn gestern. (wollen)

Ich wollte ihn gestern besuchen.

1. Wir bezahlen die Rechnung. (können)

2. Sie ging zur Bank. (müssen)

3. Kamen Sie mit? (dürfen)

4. Sie kauft einen neuen Wagen. (wollen)

5. Sie machten es gestern. (sollen)

Kopieren Sie Ihre Antworten und schicken Sie sie mit mailto:pgolz@uvic.ca

(Aus: http: / / castle.uvic.ca/german/ other_ exercises.html)

\section{Abb. 7 Name:}

Möchtest du Vokabular zum Thema Essen und Trinken / Einkaufen lernen und festigen? - Dann kannst du im Internet den Service travlang nutzen.

Besuche im Internet die Adresse http:// www.travlang.com, wähle Foreign languages for travelers.

Markiere text only, damit du nicht so lange warten mußt, und wähle Ausgangssprache (yo hablo español) und Zielsprache (deutsch).

1. Welche Themen kannst du bearbeiten?

\begin{tabular}{|l|l|}
\hline 1. & \\
\hline 2. & \\
\hline$\cdots$ & \\
\hline
\end{tabular}

Wähle das Thema Einkaufen / Essen und wähle presente un examen!

Du findest eine Liste mit $\square$ spanischen Wörtern und sollst die deutschen Äquivalente finden. Das funktioniert so:

\begin{tabular}{|l|l|l|}
\hline \hline cerveza & & $\Downarrow$ \\
\hline
\end{tabular}

Ein Klick auf den Pfeil rechts neben den Kästchen zeigt dir eine Liste mit deutschen Wörtern und Ausdrücken. Ein Klick auf ein deutsches Wort fügt es als Äquivalent neben das spanische Wort ein. Wenn du alle Aufgaben gelöst hast, kannst du sehen, welche richtig sind. Klicke auf compare sus respuestas. Neben den richtigen Lösungen erscheint ein
Die anderen Lösungen kannst du so oft korrigieren, bis alle richtig sind. Dann kannst du das nächste Quiz machen.

2. Bearbeite dieses Mal 3 Quiz und schreibe deine Resultate in die Tabelle:

\begin{tabular}{||l|l|l|l||}
\hline \hline & $\begin{array}{l}\text { Quiz } \\
\text { No.1 }\end{array}$ & $\begin{array}{l}\text { Quiz } \\
\text { No.2 }\end{array}$ & $\begin{array}{l}\text { Quiz } \\
\text { No.3 }\end{array}$ \\
\hline beim 1. Mal richtig & & & \\
\hline $\begin{array}{l}\text { Versuche, bis alles } \\
\text { richtig war }\end{array}$ & & & \\
\hline
\end{tabular}

3. Gehe nun zurück zu Deutsch und lies die Wortliste im 2.Teil. Suche in der Liste $\mathbf{1 0}$ Wörter (Ausdrücke), die du noch nicht kennst und die du gerne lernen möchtest. Schreibe diese Wörter hier auf:

\begin{tabular}{|l|l|l||}
\hline \hline & spanisch & deutsch \\
\hline 1 & & \\
\hline 2 & & \\
\hline$\cdots$ & & \\
\hline
\end{tabular}

4. Welche Wörter oder Ausdrücke aus dem Bereich Essen / Einkaufen stehen nicht in der Liste, sind aber wichtig für dich? (max. 9). Schreibe sie hier bitte auf (auf Spanisch natürlich...)

1)

2)

(...)

5. Und jetzt bitte dein Kommentar: Ich finde travlang

nützlich $\square$

unnütz $\square$, denn

interessant $\square$

langweilig $\square$ und:

6. Wenn du travlang nützlich und interessant findest, bearbeite bitte alle zwei Tage 3 Quiz und notiere deine Ergebnisse wie oben. Gib diese »Statistik « bitte ab, wenn du denkst, es ist genug.

7. Welchen Themenbereich möchtest du dann als nächsten bearbeiten? 